\title{
ULTRA-SHORT LIVED NON-RYDBERG DOUBLY EXCITED RESONANCES OBSERVED IN MOLECULAR PHOTOIONIZATION OF CO AND $\mathrm{N}_{2}$ MOLECULES
}

\author{
P. Erman, A. Karawajczyk, U. Köble, E. Rachlew, M. Stankiewicz*, \\ K. YOSHIKI-FRANZEN
}

Department of Physics I, Royal Institute of Technology, 10044 Stockholm, Sweden

\author{
AND L. VESETH
}

Department of Physics, University of Oslo, 0316 Oslo, Norway

\begin{abstract}
Our group has recently carried out measurements of dispersed fluorescence from photo-excited $\mathrm{N}_{2}$ and $\mathrm{CO}$ molecules in 19-34 eV excitation energy range using the Swedish synchrotron radiation facility MAX in Lund. The experimental results show that relative intensities of the fluorescence from different vibrational levels of the excited $\mathrm{N}_{2}^{+}$and $\mathrm{CO}^{+}$vary with the excitation energy. We associate this phenomenon with the existence of doubly excited states in $\mathrm{N}_{2}$ and $\mathrm{CO}$ in the corresponding energy regions. Our results show that the widths of the newly revealed states correspond to their lifetimes of the order of few femtoseconds.
\end{abstract}

PACS numbers: 33.80.Eh

\section{Introduction}

The study of molecular photoionization provides valuable information about the correlation of electronic and nuclear motion which is of great interest for the understanding of mechanisms of the ion formation [1]. For this reason it is essential that the experiments, which investigate the ionization dynamics in molecular systems selectively sample alternative vibrational levels of the photoion, since different vibrational levels probe alternatively internuclear configurations. Therefore requirement for vibrationally resolved measurements is a strong motivation for dispersed fluorescence experiments, since they can provide highly resolved data over a wide energy range of incident photon energies.

*Permanent address: Institute of Physics, Jagiellonian University, Reymonta 4, 30-059 Kraków, Poland. 
One can distinguish two main scenaria governing creation of a radiatively decaying excited molecular ion by photon impact

$$
\begin{aligned}
& A B+h \nu_{\mathrm{e}} \rightarrow\left(A B^{+}\right)^{*}+\mathrm{e}^{-} \rightarrow A B^{+}+h \nu_{\mathrm{f}}, \\
& A B+h \nu_{\mathrm{e}} \rightarrow A B^{*} \rightarrow\left(A B^{+}\right)^{*}+\mathrm{e}^{-} \rightarrow A B^{+}+h \nu_{\mathrm{f}},
\end{aligned}
$$

where $A B-$ an investigated molecule, $h \nu_{\mathrm{e}}-$ excitation energy and $h \nu_{\mathrm{f}}-$ fluorescence energy.

In the case of a direct photoionization (1) the populations of the vibrational final states $\left(A B^{+}\right)^{*}$ are mostly governed by the value of the Franck-Condon factors between the initial and the final states. Thus, the intensity of the fluorescence originating from the radiative decay of specific vibrational levels of $\left(A B^{+}\right)^{*}$ is proportional to the square of the overlap matrix element between the vibrational wave functions of these states and the neutral ground state of the molecule. Under these circumstances the variation of the excitation energy above the corresponding thresholds does not affect relative intensities of the vibrational lines.

On the contrary, in the case of an indirect process, when the photoionization can take place via an intermediate autoionizing excited state of the neutral molecule (2), the populations of the final vibrational states can strongly be influenced by the presence of such a state. Therefore one can expect a departure from the intensities calculated using the Franck-Condon factors for the direct process alone when the energy of the exciting photon matches the position of the intermediate state. Variations in the relative intensities of the vibrational lines with the excitation energy can be expected. The magnitude of this perturbation depends on the ratio of the relative cross-sections for competing direct and indirect processes and populations of the levels involved.

Our measurement is based on the following. The intensity of the fluorescence $I_{i}$ from a particular quantum state $i$ of the photoion is proportional to its production rate, i.e. its partial photoionization cross-section $\sigma_{\mathrm{I}}$

$$
I_{i} \sim q_{i k} \sigma_{i},
$$

where $q_{i k}$ represents the Franck-Condon factor between the ionic states involved in the radiative transition.

Accordingly, relative intensities of such transitions can provide the measure of the vibrational branching ratios

$$
\sigma_{i} / \sigma_{j}=\left(I_{i} / I_{j}\right)\left(q_{j l} / q_{i k}\right) \text {. }
$$

\section{Experiment}

The experiment was performed using synchrotron radiation from the Swedish $500 \mathrm{MeV}$ storage ring MAX in Lund. The experimental arrangement is shown in Fig. 1 and has been described in detail previously [2]. Briefly, the molecules under investigation were excited by photons from the $1 \mathrm{~m}$ normal incidence monochromator equipped with a $1200 \mathrm{~g} / \mathrm{mm}$ diffraction grating situated at the beam line 52 . Excitation energy range of 36 to $62 \mathrm{~nm}$ was covered with typical resolution of $0.4 \mathrm{~nm}$. The pressure of the investigated gas in the experimental chamber was typically kept at $30 \mathrm{mTr}$ level. The emitted fluorescence was imaged onto the 


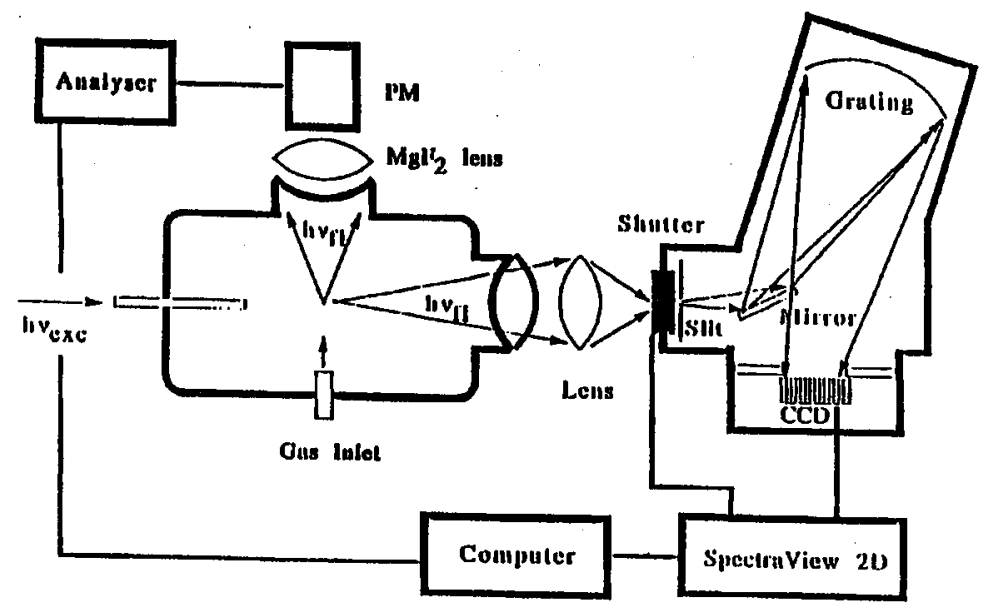

Fig. 1. The experimental setup.

entrance slit of a spectrometer covering a spectral range $190-450 \mathrm{~nm}$ with a resolution of $1.5 \mathrm{~nm}$ and the spectrum was recorded with liquid nitrogen cooled CCD multichannel array detector.

\section{Results}

\section{1. $\mathrm{N}_{2}$}

The changes in the spectrum of dispersed fluorescence originating from $B-X$ transition of $\mathrm{N}_{2}^{+}$versus different excitation energies were observed. Specifically; the $(1 ; 2)$ and $(0 ; 1)$ bands were selected for the analysis.

These measurements allow us to derive $\sigma_{1} / \sigma_{0}$ 's for the $\mathrm{N}_{2} X{ }^{1} \Sigma_{\mathrm{g}}^{+} \rightarrow$ $\mathrm{N}_{2}^{+} B^{2} \Sigma_{\mathrm{u}}^{+}$photoionization at different energies. Corresponding Franck-Condon factors were taken from Ref. [3]. The results are presented in Fig. 2. The plot shows several broad, resonant type features. They are the evidence of the existence of non-Rydberg double excited resonances (NRDERs) in $\mathrm{N}_{2}$. The widths of the resonances indicate the extremely short lifetimes of these states - in the sub-fs range. Although a very similar experiment has been performed previously [4], our data reveal much more complex structure indicating the existence of such energetic levels not seen before.

The corresponding calculations were carried out utilizing the relaxed Hartree-Fock (RHF) technique. Relaxation has been included in the computation of separate initial and final state orbitals. The calculated lifetimes are in excellent agreement with the observations. Furthermore, the positions of the calculated states agree very well with the measured $\sigma_{1} / \sigma_{0}$ extrema and are marked in Fig. 2 as $A, B, C, D$. The feature $X$ is suggested to originate from interchannel interaction with the $(3 \sigma)^{-1}$ shape resonance. The more detailed analysis of the $\mathrm{N}_{2}$ experiment can be found in Ref. [2].

\section{2. $\mathrm{CO}$}

The fluorescence from vibrational transitions $(0 ; 2),(1 ; 3)$ between $B^{2} \Sigma^{+} \rightarrow$ $X^{2} \Sigma^{+}$states of $\mathrm{CO}^{+}$were selected for the analysis. Measurements of the change 

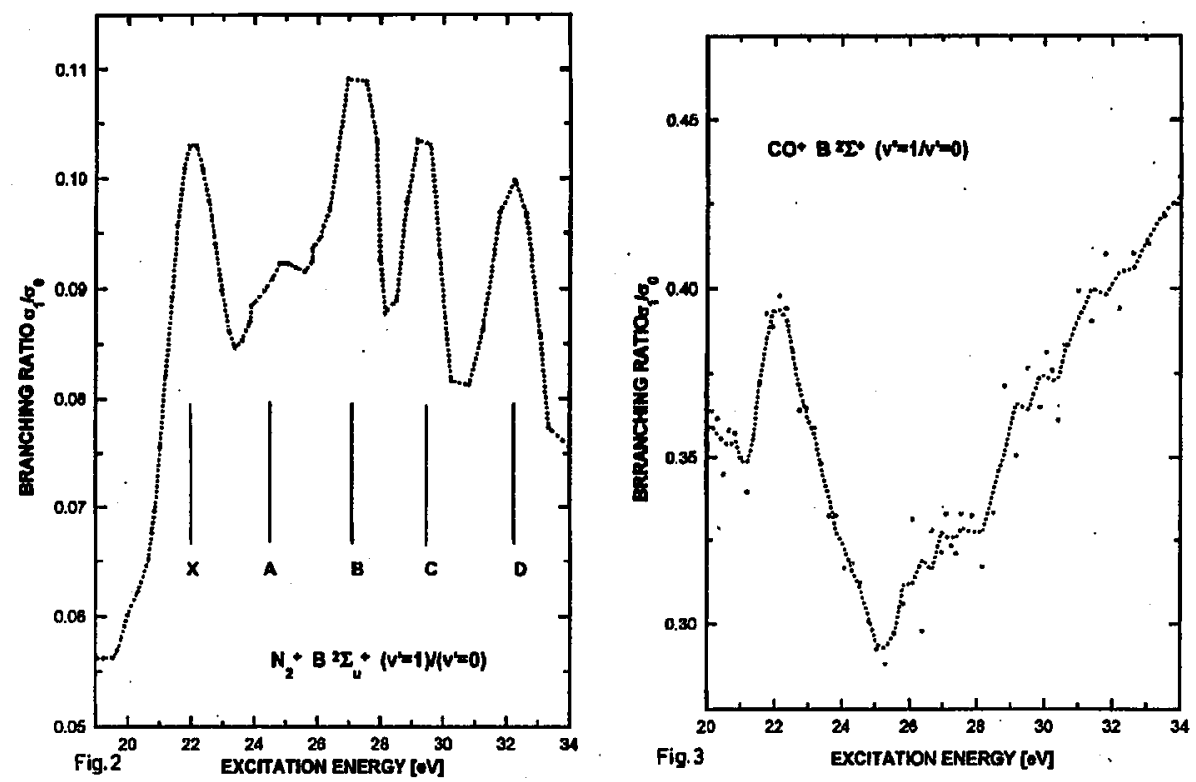

Fig. 2. Branching ratio $\sigma_{1} / \sigma_{0}$ for the $\mathrm{N}_{2}^{+} B^{2} \Sigma^{+}{ }_{u}\left(v^{\prime}=1 / v^{\prime}=0\right)$ emission following autoionization from upper doubly excited resonance in $\mathrm{N}_{2}$ (taken from [2]).

Fig. 3. Branching ratio $\sigma_{1} / \sigma_{0}$ for the $\operatorname{CO}^{+} B^{2} \Sigma^{+}\left(v^{\prime}=1 / v^{\prime}=0\right)$ emission. The variation with the energy indicates the presence of doubly excited non-Rydberg states of $\mathrm{CO}$.

in relative intensities of these lines in the excitation energy range 20-32 eV were performed.

Figure 3 presents the corresponding energy dependence of the deduced branching ratio $\sigma_{1} / \sigma_{0}$ on the energy where the relevant Franck-Condon factors were taken from Ref. [5]. Again, the variation of $\sigma_{1} / \sigma_{0}$ is pronounced and is assumed to be caused by the existence of a series of NRDER states. Preliminary theoretical calculations analogous to the ones performed for the $\mathrm{N}_{2}$ molecule confirm this hypothesis.

\section{Conclusions}

Using the dispersed fluorescence measurements presented above we were able to investigate ultra-short-lived doubly excited non-Rydberg molecular states of $\mathrm{N}_{2}$ and $\mathrm{CO}$ not detected before. Theoretical calculations confirm the existence of such states. The method opens new possibilities in spectroscopy of highly excited molecules.

\section{References}

[1] J.L. Dehmer, D. Dill, A.C. Parr, in: Photophysics and Photochemistry in Vacuum Ultraviolet, Eds. S. McGlynn, G. Findley, R. Hübner, Reidel, Dordrecht 1985, p. 341.

[2] P. Erman, A. Karawajczyk, U. Köble, E. Rachlew, K. Yoshiki-Franzén, L. Veseth, Phys. Rev. Lett. 76, 4136 (1996). 
[3] F.R. Gilmore, R.R. Laher, P.J. Espy, J. Phys. Chem. Data 21, 1005 (1992).

[4] E.D. Poliakoff, Sandeep Kakar, R.A. Rosenberg, J. Chem Phys. 96, 2740 (1992).

[5] R.W. Nicholls, Can. J. Phys. 40, 1772 (1962). 